\title{
A study on the effect of different factors on e-Commerce adoption among SMEs of Malaysia
}

\author{
Hamidreza Mahroeian*
}

${ }^{a}$ MBA (Information Technology) Graduate, Graduate School of Management, Multimedia University, Cyberjaya, Malaysia

\begin{tabular}{l}
\hline A R T I C L E I N F O \\
\hline Article history: \\
Received May 20, 2012 \\
Received in Revised form \\
July, 29, 2012 \\
Accepted 15 August 2012 \\
Available online \\
21 August 2012 \\
\hline Keywords: \\
Electronic commerce adoption \\
Small and medium enterprises \\
Perceived ease of use \\
Perceived usefulness \\
Organizational readiness
\end{tabular}

\section{Introduction}

Due to the first growth of E-commerce , companies try to create competitive advantages used of etrade in their transaction with customer (Qaemi, 2012). The Internet's World Wide Web has become the prime driver of modern E-commerce that can be an important source of competitive advantage for most business organizations, especially small and medium sized (SMEs) businesses. During the past years, IT has played an essential role om toll for communication (Chaffey et al., 2000). Internet has been one of the most popular facilities among new generation and it has been able to break the boarders among different countries (Azad \& Esmaeili, 2012). Electronic Commerce popularly known as E-Commerce has been interpreted in numerous approaches based on the main framework and also aims of the study.

\footnotetext{
* Corresponding author.

E-mail addresses: hr.mahroeian@gmail.com (H. Mahroeian) 
In this study, it has been tailored to different definitions of e-commerce in the context of B2C: "the procedure consisting of purchasing and selling of goods, services, and products with the help of electronic data transmission through the Internet and the World Wide Web known as WWW." Electronic commerce has given many advantages to both buyers and sellers; as Napier et al. (2001) addressed that by putting into practice and employing e-commerce, sellers can get the right to use and access focused markets sectors that are broadly circulated whereas purchasers can also take advantage by getting access to universal markets with different large product accessibility and different seller's variety at reasonable and reduced costs. Improving quality of product and being creative and innovative in new approaches of promoting existing available products could also be considered as important advantages (Chaudhury \& Kuilboer, 2002).

The potential advantages of e-commerce are not only assigned to the large organizations; Small and Medium Enterprises (SMEs) can also gain from electronic commerce. Moreover, it can "level the playing field" 'with big dealings, present geographical locations and time independence, and ease of communication (Chong, 2002). However, among the many advantages of e-commerce, its adoption by SMEs remains incomplete.

For instance, a survey implemented by Verizon Atlas (2003) discovered that 37\% of small businesses launched web sites principally to promote their sales and production in comparison with $9 \%$ who recognized one to sell or market online. Also in another similar study, a survey questionnaire of 444 SMEs in 2002, Pratt (2002) discovered that many SMEs were disinclined/hesitant to carry out transactions online; more than $80 \%$ were only using the Internet to communicate (via e-mail) and gather business information. Does this convey that top managers and owners of SMEs do not really appreciate the advantages and benefits of e-commerce in their businesses strategically or does this really mean that they come across major obstacles to applying it?

\section{The proposed model}

Based on the conceptual framework for this research, testable hypothesis are developed to examine whether the hypothesis formulated and developed is valid. This postulated association between variables can be then tested by using an appropriate statistical analysis method (analytical tools). The researcher will have a stronger confidence in the research done, by managing to evaluate/test and replicate the findings. Hence, the entire research project is mostly based on the theoretical framework of the research.

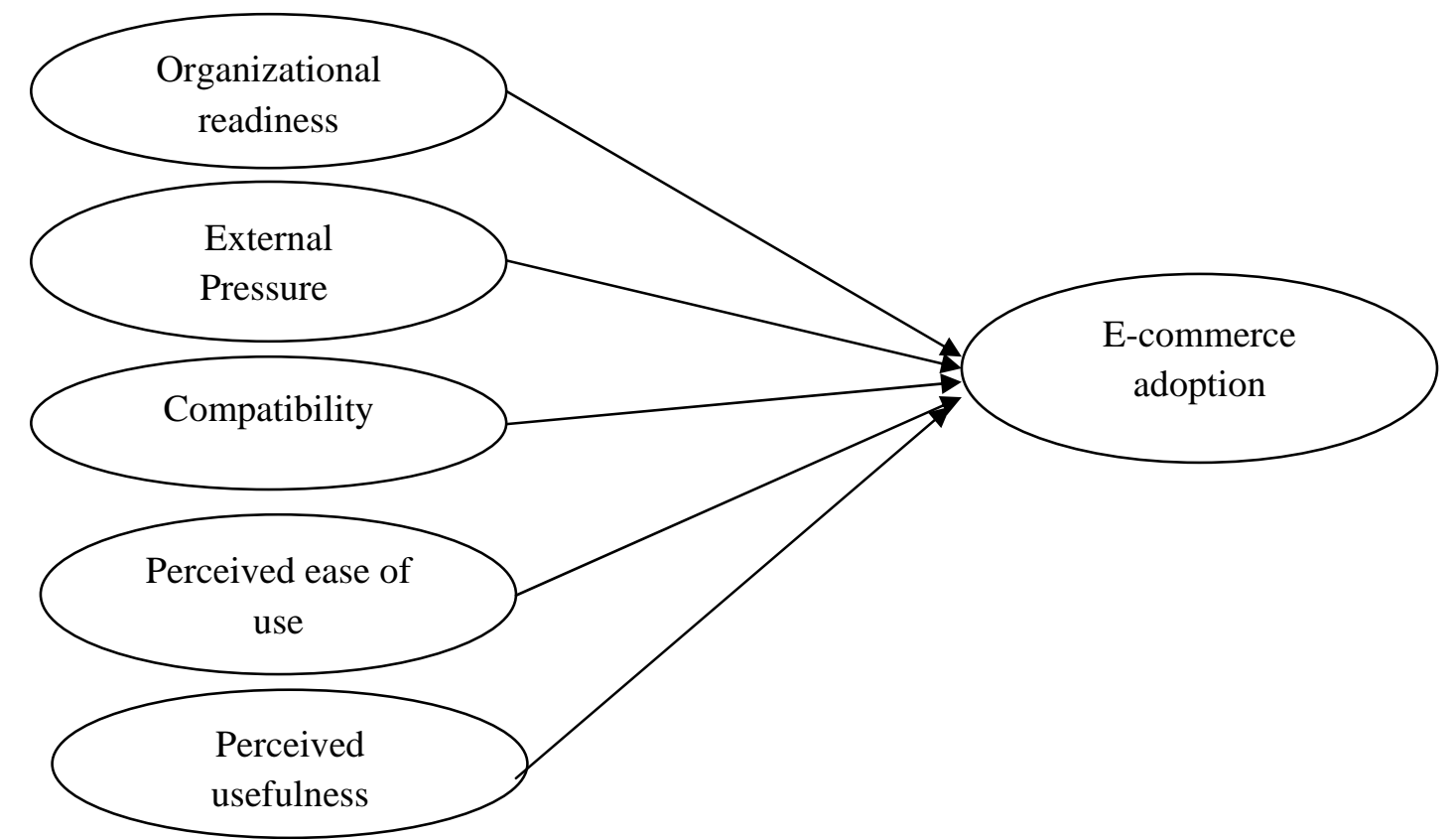

Fig. 1. Theoretical Framework 


\subsection{Hypothesis Development}

In this theoretical framework, adoption of electronic commerce is referred to as the dependent variable, while elements such as organizational readiness, compatibility, perceived ease of use (PEU), external pressure, and perceived usefulness (PU), and also perception of strategic value all are considered as independent variables. In other words, the purpose is to see whether these identified independent variables have any significant impact or influence towards the implementation of ecommerce among SMEs in Malaysian businesses.

H1: There is a direct relationship between organizational readiness and E-commerce Adoption among SMEs in Malaysia.

H2: There is an association between compatibility and adoption of E-commerce among SMEs in Malaysia.

\section{H3: External Pressure is directly correlated to E-commerce Adoption among SMEs in Malaysia.}

H4: There is a positive association between Perceived ease of Use (PEU) and E-commerce Adoption among SMEs in Malaysia.

H5: There is a correlation between Perceived Usefulness (PU) and E-commerce Adoption among SMEs in Malaysia.

\subsection{Research Gap identified}

In general, there have been a number of valuable studies and researches of E-commerce carried out by Chong (2002), Purao \& Campbell (1998), and Aldrich (1999); all of which present the importance of e-commerce and its potential advantages to organizations and small businesses. However, despite the many potential benefits of e-commerce, its implementation and adoption by SMEs remain incomplete and lagging behind. Here, the emphasis is on this "understudied" segment of business organizations where research results of large businesses cannot be generalized.

Welsh and White (1981) recognized significant differences in the financial management of small and large businesses while Ballantine et al. (1998) specified unique characteristics of SMEs such as lack of business and IT strategy, limited access to capital resources, greater emphasis on using IT and IS to automate rather than inform, influence of major customers, and limited information skills.

\subsection{Research Questions}

Given the background as illustrated in the previous section, this study seeks to answer the following research questions:

1) What are the critical factors involved in the decision made by owners and top managers or employees of SMEs for e-commerce adoption?

2) Do perceptions of strategic value of e-commerce, as observed by top managers/owners and employees of SMEs, influence their decision to adopt e-commerce?

\subsection{Objectives of the study}

The overall objective of this research is to examine the determinant factors in the adoption of ecommerce as perceived by owners and top managers of SMEs in Malaysia. Specifically, the objectives are as follows:

1) To study the perceptions of strategic value viewed by owners, influencing SMEs' top manager's decision in Malaysia. 
2) To identify and study the key factors influencing the adoption of e-commerce among SMEs in Malaysia.

\section{The results}

The results show that the major reason behind e-commerce non-adoption is that the organization is not ready to make that change because of external pressure and cultural reasons. Furthermore, organizational readiness strongly influences e-commerce implementation, and managerial support and financial, logistical, and technological factors weigh heavily in determining if an SME in Malaysia can pursue e-commerce implementation.

\subsection{Reliability test}

One of the most popular methods of reliability test is the Cronbach's Alpha and it is applied in this study to examine the reliability of the data and variables used in this research. The reliability scale was more than 0.65 and it is considered to be safe and acceptable in any topic of interest and study (Nunnally, 1978). One of the most popular methods of reliability test is the Cronbach's Alpha and it is applied in this study to examine the reliability of the data and variables used in this research. The reliability scale was more than 0.65 and it is considered to be safe and acceptable in any topic of interest and study (Nunnally, 1978).

\section{Table 1}

Reliability test

\begin{tabular}{lcc}
\hline Item & Number of Items & Cronbach's Alpha \\
\hline E-Commerce Adoption & 7 & 0.813 \\
Organizational Readiness & 3 & 0.831 \\
Compatibility & 5 & 0.734 \\
External Pressure & 5 & 0.713 \\
Ease of Use & 5 & 0.853 \\
Perceived Usefulness & 6 & 0.859 \\
\hline
\end{tabular}

As depicted in the table above, Cronbach's Alpha amount for all the independent and the only dependent variable is highly significant since all of them are above 0.65 and that shows a high consistency among the figures.

\subsection{Factor analysis}

In order to describe the most accurate correlation among all the extracted factors with original variables, factor analysis is commonly used to extract one or more factors from a large group of variables. For this study, by collecting data from 100 respondents and representing all those data in 30 different variables in order to extract 6 items which represent the model perfectly, factor analysis is used for extracting the 6 factors.

As represented in Table 2, which illustrates the result of conducting the factor analysis over 30 sets of data, the respective variables depicted are in correlation with their correspondent factor.

Therefore, based on the result provided by the factor analysis (see Table 2), we can conclude that Factor 1 is in direct correlation with Perceived usefulness and thereby Factor 1 will represent it; Factor 2 will represent organizational readiness, Factor 3 will represent ease of use, Factor 4 will represent external pressure, Factor 5 will represent compatibility, and Factor 6 will represent Ecommerce adoption. 
Table 2

Factor analysis (Rotated Component Matrix ${ }^{\mathrm{a}}$ )

\begin{tabular}{|c|c|c|c|c|c|c|}
\hline & \multicolumn{6}{|c|}{ Component } \\
\hline & 1 & 2 & 3 & 4 & 5 & 6 \\
\hline Improving job performance(PU2) & .867 & & & & & \\
\hline Usefulness in job(PU6) & .746 & & & & & \\
\hline Easy to use(EU5) & .736 & & & & & \\
\hline Accomplish specific tasks(PU1) & .723 & & & & & .308 \\
\hline Flexibility to interact with(EU2) & .630 & & .528 & & & \\
\hline Consistency(C4) & .582 & & & & & -.367 \\
\hline Becoming skillful(EU4) & .528 & .358 & .403 & & & \\
\hline Increasing productivity(PU3) & .480 & & & & & .449 \\
\hline Easier to do job(PU5) & .473 & & & & .353 & \\
\hline Technological resources(OR2) & & .758 & & & & \\
\hline Values(C2) & & .715 & & & .421 & \\
\hline Financial resources(OR1) & & .639 & .367 & & & \\
\hline Cultures(C1) & & .632 & & & .462 & \\
\hline Preferred work experience(C3) & .354 & .564 & & & & \\
\hline Easy to learn(EU1) & & & .814 & & .305 & \\
\hline Clear interaction(EU3) & & & .674 & & & \\
\hline Dependability on firm(EP3) & & & .544 & & .451 & \\
\hline Enhancing effectiveness(PU4) & .431 & & .455 & & & \\
\hline Effective support role (EC5) & & & & .756 & & \\
\hline Reap operational benefit(EC4) & & & .309 & .712 & & \\
\hline Increasing ability to compete(EC6) & & & & .667 & & \\
\hline Support linkages with suppliers(EC7) & .423 & & & .658 & & \\
\hline Social factors(EP2) & & & & & .709 & \\
\hline Industry pressure(EP4) & & & & & .691 & \\
\hline Government pressure(EP5) & & & & .321 & .482 & \\
\hline Competition factor(EP1) & .426 & & & & .441 & \\
\hline Management enthusiastic(C5) & & & .382 & & .412 & \\
\hline Reduce costs(EC1) & & & & & & .774 \\
\hline Improve customer services(EC2) & .402 & & & .400 & & .566 \\
\hline Improve distribution channel(EC3) & & & & .517 & & .540 \\
\hline
\end{tabular}

Extraction Method: Principal Component Analysis, Rotation Method: Varimax with Kaiser Normalization, a.

Rotation converged in 14 iterations.

\subsection{Hypothesis testing}

The association between the main variables is presented in this part, which includes organizational readiness, external pressure, compatibility, ease of use and perceived usefulness.

Table 3

Model Summary

\begin{tabular}{lllll}
\cline { 2 - 5 } Model & $\mathrm{R}$ & $\mathrm{R}^{2}$ & Adjusted $\mathrm{R}^{2}$ & Std. Error of the Estimate \\
\hline 1 & $.624^{\mathrm{a}}$ & .589 & .411 & .43570 \\
\hline
\end{tabular}


In order to examine the above hypothesis, multiple-regression was used. The multiple regressions analysis is the least square estimator of a linear regression model with a multi predictor variable. It is a model that relates a dependent (outcome) variable to several independent (predictor) variables. In this model, R-square is the square of the correlation coefficient between the observed value of the dependent variable and the predictor variables. R-square varies from a low of 0.00 (indicating that none of the variance can be explained), to a high of +1.0 (indicating that all of the variance can be explained).

As it is depicted in Table 3 model summery is being analyzed and tested. After data being gathered and tested, $\mathrm{R}$ is equal to 0.624 , which is $62.4 \%$ whereas $\mathrm{R}$ square value is equal to 0.589 , which is $58.9 \%$ implying that the model for this gathered data is valid, which can be interpreted as 58.9 percentages of changes in e-commerce adoption is due to changes in all independent factors.

$\mathrm{R}$ is the square root of R-Squared and is the correlation between the observed and predicted values of dependent variable (E-commerce adoption).R-Square - This is the proportion of variance in the dependent variable (E-commer adoption) which can be explained by the independent variables (Organizational readiness, compatibility, external pressure, perceived ease of use and perceived usfulness). This is an overall measure of the strength of association and does not reflect the extent to which any particular independent variable is associated with the dependent variable.

\section{Table 4}

The results of ANOVA test

\begin{tabular}{lllllll}
\hline Model & & Sum of Squares & Df & Mean Square & F & Sig. \\
\hline 1 & Regression & 11.478 & 5 & 2.296 & 9.060 & $.000^{\mathrm{a}}$ \\
& Residual & 23.819 & 94 & .253 & & \\
& Total & 35.297 & 99 & & \\
\hline
\end{tabular}

a. Predictors: (Constant), PerceiveUse, OrgRed, ExtPressure, Comp, EaseOfUse, b. Dependent Variable: Ecommerce

According to the results of ANOVA test presented in Table 4, Regression, Residual, Total - Looking at the breakdown of variance in the outcome variable, these are the categories we will examine: Regression, Residual, and Total. The Total variance is partitioned into the variance which can be explained by the independent variables (Model) and the variance which is not explained by the independent variables (Error). Sum of Squares - These are the Sum of Squares associated with the three sources of variance, Total, Model and Residual. The Total variance is partitioned into the variance which can be explained by the independent variables (Regression) and the variance which is not explained by the independent variables (Residual). $d f$ - These are the degrees of freedom associated with the sources of variance. The total variance has $\mathrm{N}-1$ degrees of freedom. The Regression degrees of freedom corresponds to the number of coefficients estimated minus 1. Including the intercept, there are 6 coefficients, so the model has $6-1=5$ degrees of freedom. The Error degrees of freedom is the DF total minus the DF model, $99-5=94$. Mean Square - These are the Mean Squares, the Sum of Squares divided by their respective DF. F and Sig. - This is the Fstatistic the p-value associated with it. The F-statistic is the Mean Square (Regression) divided by the Mean Square (Residual): 2.296/0.253 = 9.06.. The p-value is compared to some alpha level in testing the null hypothesis that all of the model coefficients are 0.

P-value was calculated to be 0.000 , which is less than 0.05 , referring to the fact that among all those variables that serve as independent factors, there must be at least one variable that can successfully predict the dependent variable. The F value examines how well the regression model fits the data. As represented in the above table 3.1.4, the computed F statistic was measured to be 9.060 indicating that $\mathrm{F}$ is significant. 
Table 5

The results of regression function ${ }^{\mathrm{a}}$

\begin{tabular}{|c|c|c|c|c|c|}
\hline \multirow[b]{2}{*}{ Model } & \multicolumn{2}{|c|}{ Unstandardized Coefficients } & \multicolumn{2}{|c|}{ Standardized Coefficients } & \multirow[b]{2}{*}{ Sig. } \\
\hline & $\mathrm{B}$ & Std. Error & Beta & $\mathrm{t}$ & \\
\hline 1 (Constant) & 2.219 & .562 & & 3.950 & .000 \\
\hline OrgRed & .192 & .080 & .252 & 2.413 & .018 \\
\hline Comp & -.077 & .113 & -.080 & -.686 & .495 \\
\hline ExtPressure & -.074 & .102 & -.075 & -.723 & .471 \\
\hline EaseOfUse & .233 & .119 & .261 & 1.956 & .013 \\
\hline PerceiveUse & .268 & .123 & .267 & 2.183 & .032 \\
\hline
\end{tabular}

a. Dependent Variable: Ecommerce

The model column shows the predictor variables (constant, OrgRed, Comp, ExtPressure, EaseOfUse, PerceiveUse). The first variable (constant) represents the constant, also referred to in textbooks as the $\mathrm{Y}$ intercept, the height of the regression line when it crosses the $\mathrm{Y}$ axis. In other words, this is the predicted value of E-commerce adoption when all other variables are 0 .

Ypredicted $=b 0+b 1 * x 1+b 2 * x 2+b 3 * x 3+b 4 * x 4+b 5 * x 5$

Ye-commerce adoption $=2.219+0.192 *$ OrgRed $-0.77 *$ Comp $-0.074 *$ ExtPressure + $0.233 *$ EaseOfUse + ).268*PerceiveUse

The column of estimates provides the values for b0, b1, b2, b3, b4 and b5 for this equation.

OrgRed - The coefficient for OrgRed is .192. So for every unit increase in OrgRed, a 0.192 unit increase in E-comerce adoption is predicted, holding all other variables constant.

Comp - For every unit increase in Comp, we expect a -0.077 unit decrease in the E-commerce adoption, holding all other variables constant.

ExtPressure - The coefficient for ExtPressure is -0.074. So for every unit decrease in ExtPressure, we expect an approximately 0.074 point increase in the E-commerce adoption, holding all other variables constant.

EaseOfUse - The coefficient for EaseOfUse is .233. So for every unit increase in EaseOfUse, we expect a .23 point increase in the E-commerce Adoption.

PerceievUse - The coefficient for PerceievUse is .268. So for every unit increase in PerceievUse, we expect a .26 point increase in the E-commerce Adoption.

$t$ and Sig. - These are the t-statistics and their associated 2-tailed p-values used in testing whether a given coefficient is significantly different from zero. Using an alpha of 0.05 :

The coefficient for OrgRed (0.192) is significantly different from 0 because its p-value is 0.018 , which is smaller than 0.05 .

The coefficient for Comp (-0.077) is not significantly different from 0 because its p-value is 0.495 , which is larger than 0.05 .

The coefficient for ExtPressure (-0.074) is not statistically significantly different from 0 because its p-value which is ).471 is definitely larger than 0.05 .

The coefficient for EaseOfUse (0.233) is statistically significant because its p-value of 0.013 is less than .05 . 
The coefficient for PerceieveUse (0.268) is statistically significant because its p-value of 0.032 is less than .05 . The intercept is significantly different from 0 at the 0.05 alpha level.

H1: There is a positive direct relationship between organizational readiness and E-commerce adoption among SMEs in Malaysia-supported.

Organizational readiness can be evaluated from two different perspectives such as technologically and financially in the organization. The term technological readiness is mostly referred to the level of superiority of information technology usage and IT management in an enterprise. On the other hand, financial readiness is concerned with the financial resources available for IT to pay for the costs of installation and the implementation of any enhancements. The survey demonstrated that organizational readiness plays a significant role on the level of e-commerce adoption among Malaysian SMEs by having a significant positive relationship and the hypothesis is accepted.

H2: There is a relationship between compatibility and adoption of E-commerce among SMEs in Malaysia-not supported.

Grandon and Pearson (2004) discovered that the interest of top management, compatibility with the organizational work environment, perceived relative advantages from e-commerce, and the employee's knowledge about computers in the company were considered as the most important elements, which would make a distinguish between the main adopters and the non-adopters of ecommerce among SMEs. The survey showed that compatibility has a significant negative relationship with e-commerce adoption among SMEs in Malaysia and this proposed hypothesis is rejected.

H3: External Pressure is not directly correlated to E-commerce Adoption among SMEs in Malaysianot supported.

Based on Iacovou's opinion in 1995, external pressure is concerned with influences from the organizational environment. This external pressure has been measured by integrating five items namely competition, dependency on other organizations applying e-commerce, the industry, social factors, and also the government. The conducted survey demonstrated that external pressure has a significant negative relationship with e-commerce adoption among Malaysian SMEs and this hypothesis is rejected.

H4: There is a positive association between Perceived ease of Use and E-commerce Adoption among SMEs in Malaysia-supported.

According to Davis (1989), perceived ease of use can be assessed by specifying how exactly IT is controllable, clear, easy to learn, and understandable, flexible, easy to become skillful in and easy to use. As demonstrated in the conducted survey from respondents, it shows that ease of use has a significant positive relationship with e-commerce adoption and this proposed hypothesis is well accepted.

H5: There is a positive correlation between Perceived Usefulness and E-commerce Adoption among SMEs in Malaysia-supported.

The definition of perceived usefulness is followed from the interpretation of the term "useful" which signifies capability of being used usefully. Perceived usefulness can be assessed by discovering the influences of IT on job performance, speed of work, effectiveness, increased productivity, making job useful and easier. The survey proved that there is a considerable positive relationship between perceived usefulness and e-commerce adoption among Malaysian SMEs and this hypothesis is accepted.

All the above objectives were formalized to the hypotheses and finally analyzed using SPSS 17.0 software and arrived at the conclusions as explained above under each proposed hypothesis. 
The results show that the major reason behind e-commerce non-adoption is that the organization is not ready to make that change because of external pressure and cultural reasons. Furthermore, organizational readiness strongly influences e-commerce implementation, and managerial support and financial, logistical, and technological factors weigh heavily in determining if an SME in Malaysia can pursue e-commerce implementation. We believe that the e-commerce adoption model in this study will have both managerial implication for practitioners in Malaysia, as well as those in other developing countries, and academic implications for researchers who are interested in ecommerce adoption in developing countries.

\section{Conclusion}

The data for this research study includes top managers/owners as well as employees of targeted small and medium enterprises in Malaysia. Data was gathered from 135 respondents from 11 small and medium enterprises in Cyberjaya. After excluding data which was not precise enough and incomplete survey questionnaires, 100 survey forms were analyzed. The model and developed hypotheses were proposed and evaluated using factor analysis and statistical package for social science or popularly known as SPSS techniques.

Based on the data gathered and analyzed results from respondents, using factor analysis method and multiple regression with the help of SPSS software, we can conclude that there is a positive relationship between factors like organizational readiness, perceived ease of use (PEU), and perceived usefulness to the adoption of e-commerce. However, there is a negative relationship found between external pressure and compatibility to e-commerce adoption.

\section{References}

Aldrich, D. F. (2009). Mastering the Digital Marketplace: Practical Strategies for Competitiveness in the New Economy. John Wiley \& Sons Inc.

Azad, N., \& Esmaeili, A. (2012). A survey on measuring the effect of website on customer satisfaction. Management Science Letters, 2(5),1639-1644.

Ballantine, J., Levy, M., \& Powel, P. (1998). Evaluating information systems in small and mediumsized enterprises: issues and evidence. European Journal of Information Systems. 7, 241-251.

Chaudhury, A., \& Kuilboer, J. P. ( 2002). E-Business and E-Commerce Infrastructure. McGraw-Hill, Boston, MA.

Chaffey, D., Mayer, R., Johnston, K., Ellis-Chadwick, F. (2000). Internet Marketing, Strategy, Implementation and Practice, FT/Prentice Hall.

Chong. (2002). Model of factors on EC adoption and diffusion in SMEs, in: Proceedings of the 1st We-B Conference. Working for E-Business: Challenges of the New e-Conomy. Fremantle,Western Australia.

Davis, F. D. (1989). Perceived usefulness, perceived ease of use, and user acceptance of information technology. MIS quarterly, 319-340.

Grandon, E., \& Pearson, J. M. (2004). E-commerce adoption: perceptions of managers/owners of small and medium sized firms in Chile. Communications of the Association for Information Systems, 13, 81-102.

Iacovou, A.L., Benbasat, I., \& Dexter., A. (1995). Electronic data interchange and small organizations: adoption and impact of technology. MIS Quarterly, 465-485.

Napier, H.A., Judd, P.J., Rivers, O.N., \& Wagner, S. W. (2001). Creating a Winning E-Business. Course Technology, Boston, MA,

Nunnally, J. C. (1978). Psychometric theory (2nd ed.). New York: McGraw-Hill.

Pratt, J. H. (2002). E-Bisiness: strategies for small business success. US SBA Office of Advocacy.

Purao, S. \& Campbell, S. (1998). Critical concerns for small business electronic commerce: some reflections based on interviews of small business owners. 
Qaemi, V. (2012). Surveying the impact of satisfaction and e-reliability on customers' loyalty in epurchase process: a case in Pars Khodro co. Management Science letters, 2(6), 2103-2112.

Welsh, J.A., \& White., J. F. (1981). A small business is not a little big business. Harvard Business Review, 18-32. 